\title{
Prospect for detecting squeezed states of light created by a single atom in free space
}

\author{
Magdalena Stobińska ${ }^{\mathrm{a}, \mathrm{b}}$, Markus Sondermann $^{\mathrm{a}}$, Gerd Leuchs $^{\mathrm{a}}$ \\ ${ }^{a}$ Institute for Optics, Information and Photonics, Erlangen-Nürnberg University, \\ Staudtstr. 7/B2, 91058 Erlangen, Germany, \\ and Max Planck Institute for the Science of Light, Günter-Scharowsky-Str. 1, Bau 24, \\ 91058 Erlangen, Germany \\ ${ }^{b}$ Institute of Theoretical Physics and Astrophysics, University of Gdańsk, Poland
}

\begin{abstract}
We discuss the possibilities of studying in detail the dynamics of spontaneous emission of a single photon by a single atom and measuring the transient degree of squeezing by means of full solid angle fluorescence detection.
\end{abstract}

Key words: squeezing, resonance fluorescence, single photon, single atom

\section{Introduction}

We are mourning our teacher and colleague Krzysztof Wódkiewicz whom we will fondly remember as a skillful scientist, sharp thinker and friend.

Quadrature squeezed states of light are a basic resource for continuous variable $(\mathrm{CV})$ based quantum communication. Two independent $\mathrm{CV}$ squeezed states interfering on a beam splitter form at its output CV EinsteinPodolsky-Rosen entangled states [1, 2]. Experimentally this entanglement was demonstrated for vacuum states [3] and for intense laser beams [4]. Based on squeezed-state entanglement quantum teleportation of coherent states was experimentally achieved [5]. Recently it was shown that a single quadrature squeezed photon also enables obtaining teleportation of coherent state qubits [6]. Moreover, quadrature squeezing can be transformed into polarization squeezing [7]. CV squeezed states have also been useful

Email address: magda.stobinska@mpl.mpg.de (Magdalena Stobińska) 
for developing various other tools for quantum information processing. For example, they have been used for realizing quantum nondemolition coupling and thus CV quantum erasing [8]. Furthermore, a method for dense quantum coding for the quadrature amplitudes of the electromagnetic field has been proposed [9].

Whereas in the majority of the references mentioned so far squeezed states have been used as a tool, we want to focus on a more fundamental aspect here. We propose an experiment that is expected to enable the successful observation of a squeezed state that is generated in one of the most fundamental settings of quantum optics: the spontaneous emission of a single photon by a single two-level atom that is prepared in a suitable superposition state.

The name 'squeezed' states emerges from their property that the quantum uncertainty in one of two noncommuting observables $[A, B]=i C$ in these states is decreased $(\Delta A)^{2}<\frac{1}{2}|\langle C\rangle|$ at the cost of increasing uncertainty in the other one $(\Delta B)^{2}>\frac{1}{2}|\langle C\rangle|$, in order to obey the Heisenberg uncertainty relation $\Delta A \Delta B \geq \frac{1}{2}|\langle C\rangle|$. Amplitude and phase quadrature operators are defined as

$$
X_{1}=1 / 2\left(a+a^{\dagger}\right), \quad X_{2}=i / 2\left(a^{\dagger}-a\right),
$$

where $a$ and $a^{\dagger}$ denote annihilation and creation operators respectively, $X_{1,2}$ are noncommuting observables $\left[X_{1}, X_{2}\right]=i / 2$ for which $\Delta X_{1} \Delta X_{2} \geq 1 / 4$. Therefore, a state for which $\left(\Delta X_{k}\right)^{2}<1 / 4$ for $k=1,2$ is called quadrature squeezed.

Usually quadrature squeezed states are produced via a squeezing transformation generated by a Hamiltonian quadratic in annihilation $a$ and creation $a^{\dagger}$ operators applied to a coherent state $|\alpha\rangle$. For a single mode of light the transformation takes the following form

$$
\hat{S}=\exp \left\{\frac{\xi^{*}}{2} a^{2}-\frac{\xi}{2} a^{\dagger^{2}}\right\},
$$

where $\xi$ is called the squeezing parameter.

Early on it was realized that applying the operator $\hat{S}$ to the vacuum state leads to a superposition of even number Fock states [10], hence the name two-photon coherent states for this special class of squeezed states. In experiments squeezing is realized either in the parametric amplification or in four wave mixing process in $\chi^{(3)}$ nonlinear medium (e.g. in optical fiber).

Squeezing is present not only in a superposition of two macroscopic coherent states [11] but also in the superposition of vacuum and a single photon 
arising in the process of spontaneous emission of an atom into a single cavity mode [12]. At the time this came a bit as a surprised.

Several years later, different schemes for the generation of arbitrary quantum states of light - including the superposition states mentioned above have been proposed [13, 14, 15, 16]. All these schemes have in common that they are based on the interaction of atoms with the single-mode light field of a cavity. However, here we concentrate on the interaction dynamics of light and single atoms in free space, in particular in the dynamics of spontaneous emission. In other words, the squeezing in the fluorescence of a single atom [17, 18] is a sensitive tool for studying the dynamics of spontaneous emission in an unprecedented way. Therefore, we sacrifice the possibility of generating arbitrary quantum states and restrict the discussion to the most simple superposition state, namely the one described in Ref. [12]. As we will outline below, this state can be generated by preparing a single two-level atom in the corresponding superposition of its ground state and its excited state and full solid angle collection of the spontaneously emitted photon.

The paper is organized as follows. In section 2 we summarize the theoretical model for obtaining the single photon squeezed state put forward in Ref. [12]. Section 3 is devoted to the discussion about the possibilities for realization and detection of squeezing in superposition states in a suitable free space experimental setup.

\section{Model}

According to the squeezing transformation in Eq. (21), the squeezed vacuum state is a superposition of even Fock number states [10]

$$
\hat{S}|0\rangle=e^{-\frac{1}{2} \ln \operatorname{ch} \xi} \exp ^{-\frac{\operatorname{th} \xi}{2} a^{\dagger^{2}}}|0\rangle=e^{-\frac{1}{2} \ln \operatorname{ch} \xi}\left\{|0\rangle-\sqrt{2 !} \frac{\operatorname{th} \xi}{2}|2\rangle+\sqrt{4 !} \frac{\operatorname{th}^{2} \xi}{4 \cdot 2 !}|4\rangle+\ldots\right\},
$$

where the squeezing parameter $\xi$ was assumed to be real for simplicity. Wódkiewicz et al. [12] discovered that in one photon superposition states

$$
|\varphi\rangle=\gamma|0\rangle+\beta|1\rangle
$$

\footnotetext{
${ }^{1}$ One of us (GL) vividly remembers Krzysztof Wódkiewicz coming to his office at the Max Planck Institute for Quantum Optics in Garching emphasizing that he had just found an unexpected result: The superposition of the $|0\rangle$ and the $|1\rangle$ Fock states may show squeezing. The surprise was that squeezed states generated from vacuum were not exclusively superpositions of even Fock states.
} 


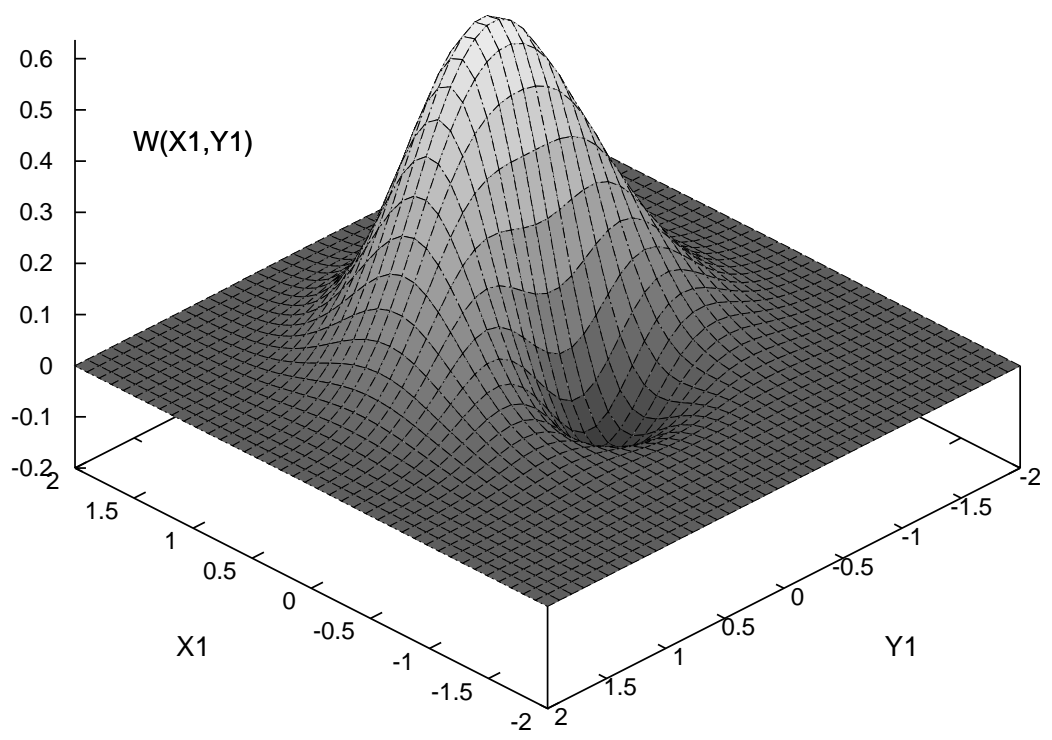

Figure 1: Wigner function $W\left(X_{1}, X_{2}\right)$ evaluated for the one-photon superposition state in Eq. (41) for $\gamma=\sqrt{2 / 3}$ and $\beta=\sqrt{1 / 3}$.

where $|\gamma|^{2}+|\beta|^{2}=1$, quadrature squeezing is present as well for $|\beta|<$ $1 / \sqrt{2}$ and some special values of relative phase $\phi$ between the probabilities amplitudes

$$
\left(\Delta X_{1}\right)^{2}(\phi=0, \pi)=\left(\Delta X_{2}\right)^{2}(\pi / 2,3 \pi / 2)=1 / 4+|\beta|^{2}\left(|\beta|^{2}-1 / 2\right) .
$$

The values of $\phi=0, \pi(\pi / 2,3 \pi / 2)$ correspond to the amplitude (phase) quadrature squeezing respectively. The state Eq. (4) is presented in terms of a Wigner function in Fig. 1 for $\gamma=\sqrt{2 / 3}$ and $\beta=\sqrt{1 / 3}$.

The superposition given in Eq. (4) arises naturally during interaction of a single quantized electromagnetic field mode initially in the vacuum state with a two-level atom initially in a superposition of excited and ground states. The system is described by the Jaynes-Cummings Hamiltonian

$$
H=1 / 2 \hbar \omega_{0} \sigma_{z}+\hbar \omega a^{\dagger} a+\hbar \lambda\left(a^{\dagger} \sigma_{-}+\sigma_{+} a\right)
$$

where $\omega_{0}$ is the atomic transition frequency, $\sigma_{z}$ is the inversion operator, $\omega$ is the field mode frequency, $a$ and $a^{\dagger}$ are field annihilation and creation operators, $\lambda$ is interaction strength, $\sigma_{-}$and $\sigma_{+}$are lowering and rising atomic operators. For the relation between the Jaynes-Cummings single mode model and the Wigner-Weisskopf model with a continuum of modes see Sec. 3 below. 
Let us consider an atom prepared in a coherent superposition of excited $|e\rangle$ and ground $|g\rangle$ states which interacts with a vacuum field $|0\rangle$

$$
|\Psi(t=0)\rangle=\cos (\theta / 2)|e, 0\rangle+e^{i \phi} \sin (\theta / 2)|g, 0\rangle .
$$

The atom can spontaneously decay to its ground state and emit a photon. In the Schrödinger picture and on resonance the total wave function of the system at time t reads

$$
\begin{aligned}
|\Psi(t)\rangle & =\cos (\theta / 2) \cos (\lambda t) e^{-i \omega t}|e, 0\rangle+e^{i \phi} \sin (\theta / 2)|g, 0\rangle \\
& -i \cos (\theta / 2) \sin (\lambda t) e^{-i \omega t}|g, 1\rangle .
\end{aligned}
$$

The uncertainties in quadrature operators for this state measured by a homodyne detector are equal to

$$
\begin{aligned}
& \left(\Delta X_{1}\right)^{2}=1 / 4+\cos ^{2}(\theta / 2) \sin ^{2}(\lambda t)\left(1 / 2-\sin ^{2} \phi \sin ^{2}(\theta / 2)\right), \\
& \left(\Delta X_{2}\right)^{2}=1 / 4+\cos ^{2}(\theta / 2) \sin ^{2}(\lambda t)\left(1 / 2-\cos ^{2} \phi \sin ^{2}(\theta / 2)\right) .
\end{aligned}
$$

The phase $\phi$ denotes the phase difference between the field and the local oscillator in homodyne detection. For $\theta=2 \pi / 3,4 \pi / 3$ and $\phi=\pi / 2$ squeezing appears in the amplitude quadrature

$$
\left(\Delta X_{1}\right)^{2}=1 / 4-1 / 16 \sin ^{2}(\lambda t) .
$$

Note that for time $t=\pi / 2 \lambda$ we obtain the desired superposition Eq. (44). Furthermore, no squeezing arises for a purely excited $(\theta=0)$ or de-excited $(\theta=\pi)$ atom.

As indicated by Eqs. (910) the amount of squeezing in the emitted photon superposition state is dependent on the state in which the atom was prepared. Thus, a precise measurement of the photonic state would give some insight into the state - possibly unknown a priori - in which the atom was before the emission process.

Moreover, in [12] a link between atomic dipole $D=|g\rangle\langle e|=D_{1}+i D_{2}$ squeezing and radiated field squeezing has been established. If the initial atomic state satisfies that $\left\langle\left[D^{\dagger}, D\right]\right\rangle<0$ and the dipole is squeezed : $\left(\Delta D_{1}\right)^{2}:<0$ the field will be squeezed as well. Since in resonance fluorescence the normally ordered variance of the electric field in the far field zone is related to the normally ordered variance of the atomic dipole, it is possible to observe the quadrature squeezing present in the one photon superposition state by means of resonance fluorescence preparing the atomic state properly. 


\section{Experimental prospects}

The maximum amount of squeezing predicted by Eqs. (5) and (11) is $1.25 \mathrm{~dB}$ for $|\beta|=1 / 2$. This small reduction of quadrature fluctuations calls for a sophisticated detection scheme.

First, the atom has to be prepared in the desired superposition state by application of a suitable optical pulse. Identifying $|\beta|$ with $|\cos (\theta / 2)|$ determines the area of the excitation pulse that prepares the atom in the superposition state that enables maximum squeezing. The state of the radiation field then has to be detected transiently during the emission process via time resolved homodyne measurements (e.g., [19, 20]).

Second, detection losses have to be minimized: The superposition state has to be collected ideally over the complete solid angle of the atomic emission, i.e., the full solid angle. Recently, a setup based on a deep parabolic mirror has been proposed that is capable of almost full solid angle coverage if the atom is located in the focus of the mirror [21, 22] (so far such a mirror does not yet exist with the required quality being essentially aberration free, but the aberrations can be corrected to a large extent by means of appropriate phase plates [23]). E.g., if one monitors the emission of a $\Delta \mathrm{m}=0$ transition ( $\pi$-polarization) by an atom with its quantization axis oriented along the optical axis of the parabolic mirror, current technology facilitates the collection of $94 \%$ of the light emitted by the atom. Thus, the full mode into which the atom emits can be detected. We intend to use the ${ }^{1} \mathrm{~S}_{0} \leftrightarrow{ }^{3} \mathrm{P}_{1}$ ground state transition of ${ }^{174} \mathrm{Yb}^{2+}$. It is planned to locate the ion in the focus of the parabolic mirror by means of a needle-like ion trap. First successful tests of such a trap geometry have been performed recently [24]. One might be concerned whether the parabolic mirror modifies the free space modes. We conjecture that a parabolic mirror with a focal length much larger than the wavelength of the atomic transition - as it is the case in the planned setup - does not change the density of modes at its focal point (see Ref. [22] for a qualitative discussion and also Ref. [25]).

Furthermore, the mode of the local oscillator employed in the homodyne measurement has to be matched to the atomic transition. For known emission characteristics, the mode after collection by the parabolic mirror can be calculated in a straightforward fashion [21]. In the case at hand, the mode profile of the $\pi$-polarized emission after reflection off the mirror has a strong overlap with radially polarized doughnut modes, which are easily produced experimentally [27]. 
At this point one might wonder whether the single mode Jaynes-Cummings model applied in the previous section is suitable for comparison with the free space setting of our experimental setup. To make the connection, note that in the best case the atom emits into a single spherical dipole mode. The problem can thus be treated by a one dimensional model 26]. One difference still remains: the free space atom emits into a continuum of frequency modes. We argue that such a superposition of pure states is itself a pure state and effectively a single pulsed temporal mode. One also might understand the mode operators $a$ and $a^{\dagger}$ as operators for modes with a certain frequency distribution, as they are employed in Ref. [28]. For a photon generated by an atom in its excited state that decays via interaction with all free space field modes this distribution is Lorentzian. The corresponding temporal envelope of the photon is exponentially decaying as expected in free space. In this sense the free space case should be comparable to the scenario discussed by Wódkiewicz et al. The experiment will show whether this equivalence holds.

In other words, unlike in the Jaynes-Cummings model where the squeezed superposition state occurs at every $t_{n}=(n+1 / 2) \pi / \lambda$ for integer $n$, the photonic superposition state propagates away from the atom after spontaneous decay. The corresponding wave packet has approximately the length of the atomic upper state lifetime. In the case of the $\mathrm{Yb}^{2+}$ transition mentioned above the life time is $230 \mathrm{~ns}$ [29]. This is beneficial for time resolved homodyne measurements, since the feasibility of this detection method has been demonstrated with optical pulses of considerably smaller duration [19].

However, one has to be aware that if the squeezed superposition state has a Lorentzian spectral distribution the local oscillator used in homodyne detection should ideally have the same spectral shape. We intend to fulfill this requirement by cutting pulses out of continuous wave (cw) laser beams by means of, e.g., acousto-optic modulators. The cw laser has to be frequency stabilized to a line width much smaller than the atomic transition line width. Then, an exponentially decaying pulse with its time constant matched to the atomic life time and a length of a few life times spectrally matches the squeezed superposition state almost perfectly. Again, we emphasize that the characteristics of the ${ }^{3} \mathrm{P}_{1}$ state of $\mathrm{Yb}^{2+}$ appears to be advantageous for this task: The atomic line width of approximately $700 \mathrm{kHz}$ requires a $\mathrm{cw}$ laser that is stabilized to a line width of about $1 \mathrm{kHz}$. The latter number is well within the scope of up-to-date technology. 


\section{Conclusions}

More than two decades ago, K. Wódkiewicz proposed to use one photon superposition states for the generation of quadrature squeezed light [12]. As outlined here, we argue that such a realization is feasible in a setup based on a single ion in free space when collecting the emitted radiation with a deep parabolic mirror. This setup has initially been intended to facilitate efficient absorption in free space [22]. But as elaborated in this paper, it can also be applied for the measurement of the transient quantum properties of the field emitted by a single atom. Hence, for the application in mind our setup poses a suitable alternative to the schemes for quantum state generation based on resonators [13, 14, 15, 16], since the latter hinder free space interaction between atom and photon by design.

\section{Acknowledgments}

M. St. is partially supported by QAP European project.

\section{References}

[1] G. Yeoman and S. M. Barnett, J. Mod. Opt. 40, 1497 (1993).

[2] G. Leuchs, T.C. Ralph, Ch. Silberhorn, and N. Korolkova, J. Mod. Opt. 46, 1927 (1999).

[3] Z. Y. Ou, S. F. Pereira, H. J. Kimble, and K. C. Peng, Phys. Rev. Lett. 68, 3663 (1992).

[4] Ch. Silberhorn, P. K. Lam, O. Weiss, F. König, N. Korolkova, and G. Leuchs, Phys. Rev. Lett. 86, 4267 (2001).

[5] A. Furusawa, J. L. Sorensen, S. L. Braunstein, C. A. Fuchs, H. J. Kimble, E. S. Polzik, Science 282, 706 (1998).

[6] A. M. Brańczyk and T. C. Ralph, Phys. Rev. A 78, 052304 (2008).

[7] J. Heersink, T. Gaber, S. Lorenz, O. Glöckl, N. Korolkova, and G. Leuchs, Phys. Rev. A 68, 013815 (2003).

[8] U. L. Andersen, O. Glöckl, S. Lorenz, G. Leuchs, and R. Filip, Phys. Rev. Lett. 93, 100403 (2004). 
[9] S. L. Braunstein and H. J. Kimble, Phys. Rev. A 61, 042302 (2000).

[10] H. P. Yuen, Phys. Rev. A 13, 2226 (1976).

[11] W. Schleich, M. Pernigo, and F. Le Kien, Phys. Rev. A 44, 2172 (1991).

[12] K. Wódkiewicz, P. L. Knight, S. J. Buckle, and S. M. Barnett, Phys. Rev. A 35, 2567 (1987).

[13] K. Vogel, V. M. Akulin, and W. P. Schleich, Phys. Rev. Lett. 71, 1816 (1993).

[14] A. S. Parkins, P. Marte, P. Zoller, and H. J. Kimble, Phys. Rev. Lett. 71, 3095 (1993).

[15] B. M. Garraway, B. Sherman, H. Moya-Cessa, P. L. Knight, and G. Kurizki, Phys. Rev. A 49, 535 (1994).

[16] C. K. Law, and J. H. Eberly, Phys. Rev. Lett. 76, 1055 (1996).

[17] D. F. Walls, and P. Zoller, Phys. Rev. Lett. 47 , 709 (1981).

[18] Z. H. Lu, S. Bali, and J. E. Thomas, Phys. Rev. Lett. 81, 3635 (1998).

[19] H. Hansen, T. Aichele, C. Hettich, P. Lodahl, A. I. Lvovsky, J. Mlynek, and S. Schiller, Opt. Lett. 26, 1714 (2001).

[20] J. Wenger, R. Tualle Brouri, and Ph. Grangier, Opt. Lett. 29, 1267 (2004).

[21] N. Lindlein, R. Maiwald, H. Konermann, M. Sondermann, U. Peschel, and G. Leuchs, Laser Physics 17, 927 (2007).

[22] M. Sondermann, R. Maiwald, H. Konermann, N. Lindlein, U. Peschel, and G. Leuchs, Appl. Phys. B 89, 489 (2007).

[23] M. Sondermann et al, unpublished (2009).

[24] R. Maiwald, D. Leibfried, J. Britton, J. C. Bergquist, G. Leuchs, and D. J. Wineland, Nature Physics 5, 551 (2009).

[25] M. Stobinska, and R. Alicki, arXiv:0905.4014 (2009). 
[26] M. Stobinska, G. Alber, and G. Leuchs, EPL 86, 14007 (2009).

[27] S. Quabis, R. Dorn, and G. Leuchs, Appl. Phys. B 81, 597 (2005).

[28] P. P. Rohde, W. Mauerer, and Ch. Silberhorn, New Journal of Physics 9, 91 (2007).

[29] Z. G. Zhang, Z. S. Li, S. Svanberg, P. Palmeri, P. Quinet, and E. Biemont, Eur. Phys. J. D 15, 301 (2001). 